\title{
Rapid prototyping for multi-application sensor networking
}

\section{Jorge Portilla, Angel De Castro, Ana Abril, and Teresa Riesgo}

Developing integrated hardware interfaces for different actuators allows rapid and easy integration into existing wireless networks.

Wireless sensor networks (WSNs) have become widely relevant in recent years, facilitating data collection through a large number of nodes measuring different physical parameters in various environments. Such networks require low power consumption in the individual sensors and adequate protocols for communication between the nodes. Effective platform hardware design is also crucial. Key features of the technology are the ubiquity of these systems, the need for transparency to the end user, and the variety of predicted applications. Because so many possible applications exist, using WSNs to their fullest potential depends on our ability to adapt new applications to existing platforms.

Rapid prototyping can assist in the process of adapting applications to current platforms. However, one of the main obstacles to this approach is that sensors have heterogeneous interfaces. Some of the numerous different transducer (sensor/actuator) interfaces currently in existence, such as the inter-integrated circuit (I2C), are very popular. However, there will probably never be a single common interface for all types of transducer because of commercial interests and special features. Therefore, when adapting a new sensor, most of the work must be done from scratch, making it difficult to develop applications quickly. This is the problem we are trying to solve.

Here we propose a very high speed integrated circuit (VHSIC) hardware description language (VHDL) library for sensor and actuator interfaces. The purpose is to have a set of interfaces that accommodate the most common sensors and actuators, enabling rapid connections to new equipment. Moreover, the concept presented here may be used for new interfaces that can be easily developed. The VHDL implementation is independent of the final platform; any field-programmable gate array (FPGA) or application-specific integrated circuit may be used. This min-
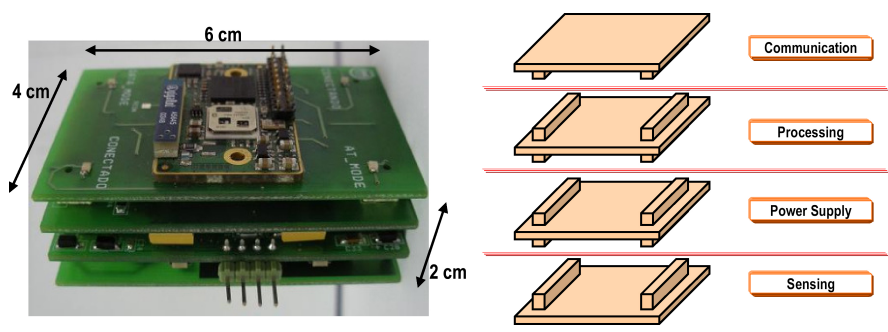

Figure 1. The four-layer architecture for sensor nodes in WSN contains layers for communication, processing, power supply, and sensing/actuating.

imizes redesign effort and makes rapid prototyping easier. The interfaces are installed in a hardware platform for sensor networks developed at our institution.

Hardware node design becomes critical in WSNs for meeting the requirements mentioned above. Modular hardware nodes are often used to make rapid prototyping easier, as well as to allow for quicker redesign and the ability to reuse some of the hardware modules. ${ }^{1-3}$ With this approach, it is easy to redesign the platform to adapt the system to different scenarios and applications. This concept was developed in our research group's previous work, and our modular platform is available for research and development. ${ }^{4}$ Figure 1 shows the four-layer platform, the main features of which are modularity, low cost, medium size, and easy adaptation to different applications.

This modular platform is divided into four functional layers: communication, processing, power supply, and sensing/actuating. The processing layer includes a microcontroller and an FPGA: this gives the platform a great deal of processing power as well as flexibility. Modularity in the hardware node must be combined with flexibility in the processing devices in order to obtain the maximum adaptability.

Continued on next page 
To simplify the connection and use of transducers, we aim to create a library with the most common interfaces for sensors, and to improve and extend this library as new transducers come on the market. Moreover, our strategy is to develop the interfaces in hardware to exploit the concurrency possibilities of the processing layer of the platform, allowing the microcontroller to perform other tasks related to the control of the global system. Figure 2 shows a diagram of the implementation strategy used for the interfaces, using our platform as the base for the WSN.

The interfaces are described in VHDL, which makes the solution independent of the final implementation as long as it is based on custom hardware. The library is composed of different modules that deal directly with every transducer (analog or digital) but work through a common interface. The transducer is connected to the corresponding module of the library, which is different for every interface, and finally this module is connected through the common interface to the rest of the circuit (see Figure 3). This type of architecture is based on the IEEE1451.2 standard. ${ }^{5}$

In particular, the different modules developed in the proposed library are used for pulse-width modulation, frequency/period modulation, I2C, Sensirion interface (similar to I2C), interface for analog transducers (module to interact with analog-to-digital

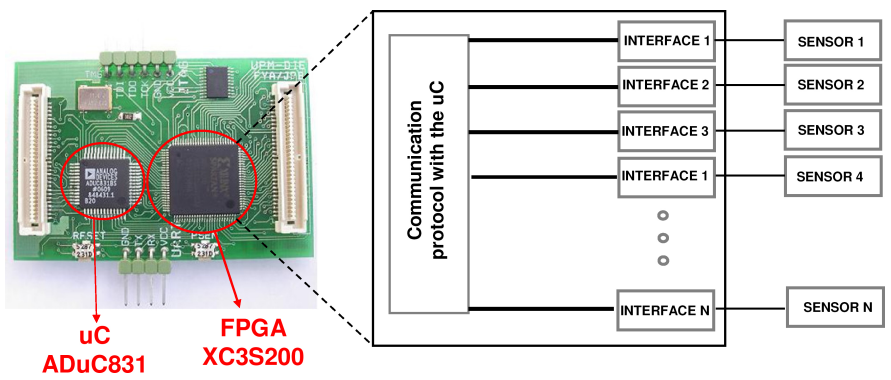

Figure 2. Interfaces for transducer control adapted to WSN platform.

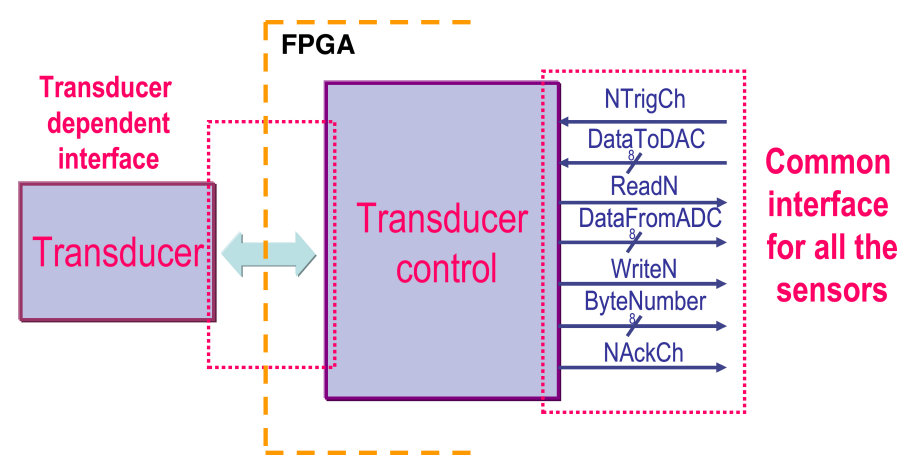

Figure 3. General structure chosen for the control of a transducer. and digital-to-analog converters), and 1-Wire ${ }^{\circledR}$. Detailed information about the interfaces is available in our paper. ${ }^{6}$

This library is used on the modular platform for WSNs, and makes the sensor almost transparent for the user, who always sees the same signals independently of the sensor being used. Exhaustive simulations have been carried out and experimental tests have also been conducted for every module in the library. The transducer interface modules, integrated into our platform, are currently being used for several applications under development in areas including the structural control of walls, the mining industry, and fruit transportation control. In addition, an entertainment application-which includes a handheld device that can be manipulated to produce musical effects-is under development. This is demonstrated in our video clip. ${ }^{7}$ (Make sure to switch your loudspeakers on!)

\section{Author Information}

\section{Jorge Portilla, Teresa Riesgo, and Ana Abril}

Center for Industrial Electronics (CEI)

Polytechnic University of Madrid (UPM)

Madrid, Spain

http:/ / www.cei.upm.es/cei_personal/Teresa.htm

http:/ / www.upmdie.upm.es/ jportill/

http:/ /www.cei.upm.es

Jorge Portilla is a teaching assistant at CEI-UPM. He has an MSc degree in physics from the Complutense University of Madrid. He is pursuing his $\mathrm{PhD}$ in wireless sensor networks.

Ana Abril is a researcher at CEI-UPM. She holds MSc and $\mathrm{PhD}$ degrees from Pierre and Marie Curie University in France. Her research interests are mainly wireless sensor networks and power modelling

Teresa Riesgo is a professor of electronics at CEI-UPM with both an $\mathrm{MSc}$ and a PhD in electrical engineering from UPM. Her research activities include wireless sensor networks, reconfigurable hardware, and power-aware design of embedded systems.

\section{Angel De Castro \\ Superior Polytechnic School \\ Autonomous University of Madrid \\ Madrid, Spain}


Dr. Angel de Castro is an associate professor at the Autonomous University of Madrid and has an MSc and PhD in electrical engineering from UPM. His research interests include digital control of power converters and wireless sensor networks

\section{References}

1. A. Y. Benbasat and J. A. Paradiso, A compact modular wireless sensor platform, Proc. Fourth IEEE Int'1 Symp. on Info. Proc. in Sensor Networks, pp. 410-415, Apr 2005. 2. L. Nachman, R. Kling, R. Adler, J. Huang, and V. Hummel, The Intel Mote Platform: A Bluetooth-based sensor network for industrial monitoring, Proc. Fourth IEEE Int'1 Symp. on Info. Processing in Sensor Networks, pp. 437-442, Apr. 2005.

3. S. Yamshita, T. Shimura, K. Aiki, K. Ara, Y. Ogata, I. Shimokawa, T. Tanaka, H. Kuriyama, K. Shimada, and K. Yano, A $15 \times 15,1 \mu$ A, reliable sensor-net module: Enabling application-specific nodes, Proc. Fifth IEEE/ACM Int'l Conf. on Info. Processing in Sensor Networks, pp. 383-390, April 2006.

4. J. Portilla, A. de Castro, E. de la Torre, and T. Riesgo, A modular architecture for nodes in wireless sensor networks, J. Universal Comp. Sci. 12 (3), pp. 328-339, March 2006.

5. IEEE 1451.2 standard for a smart transducer interface for sensors and actuators: transducer to microprocessor communication protocols and Transducer Electronic Data Sheet (TEDS) formats. 1997.

6. J. Portilla, A. de Castro, A. Abril, and T. Riesgo, Integrated hardware interfaces for modular sensor networks, Proc. SPIE 6590, May 2007. doi:10.1117/12.723773

7. Video illustrates an entertainment application with a handheld device that produces musical effects. Credit: Teresa Riesgo, Polytechnic University of Madrid. http://spie.org/documents/newsroom/videos/0851/CookiesApp.avi 